\title{
Analysis of the interbeat interval increment to detect obstructive sleep apnoea/hypopnoea
}

\author{
F. Roche*, S. Celle*, V. Pichot*, J-C. Barthélémy* and E. Sforza\#
}

ABSTRACT: The prevalence of obstructive sleep apnoea/hypopnoea syndrome (OSAHS) is underestimated and its diagnosis is costly and restricted to specialised sleep laboratories. The frequency component of interbeat interval increment (III) has been proposed as a simple and inexpensive diagnostic tool in OSAHS.

In a set of 150 patients with clinically suspected sleep-related breathing disorder, the actual predictive accuracy of the power spectral density of the III of the very low frequencies (\%VLFI) was analysed by comparing with the apnoea/hypopnoea index (AHI), as assessed by synchronised polysomnography.

OSAHS was defined in 100 patients according to an $\mathrm{AHI} \geqslant 15$ events $\cdot \mathrm{h}^{-1}$. Receiver operator characteristic curves built for \%VLFI confirmed that this variable was able to separate OSAHS positive from OSAHS negative with statistical significance. Using an appropriate threshold ( $>4 \%)$, \%VLFI demonstrated a positive predictive value of $80 \%$. Misclassification of false-positive subjects occurred when the patient presented significant sleep discontinuity and sleep fragmentation (sleep fragmentation index $\geqslant 50$ events $\cdot h^{-1}$ ) related to insomnia or periodic limb movements.

A power spectral density of the interbeat interval increment of very low frequencies $>4 \%$ allowed correct classification of obstructive sleep apnoea/hypopnoea syndrome when the clinical history suggested sleep-related breathing disorders and when moderate-to-severe cases are considered. Higher power spectral density of the interbeat interval increment of very low frequencies may also indicate disrupted sleep in the absence of clear clinical symptoms of sleep apnoea/hypopnoea syndrome.

KEYWORDS: Autonomic nervous system activity, ECG Holter, heart rate variability, sleep apnoea/ hypopnoea syndrome, sleep fragmentation

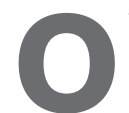

bstructive sleep apnoea syndrome (OSAS) is a common, prevalent and probably underestimated disorder [1], characterised by repetitive collapse of the upper airway during sleep, with resulting hypoxaemia, surges in sympathetic activity with arousals and cumulative sleep fragmentation. OSAS has been associated with neuropsychological impairment, increased risk of vehicular accidents [2] and cardiovascular consequences, such as hypertension, myocardial infarction, stroke and increased mortality rates $[3,4]$.

Since changes in the autonomic nervous system have been described during apnoea, as reflected by increased heart rate variability (HRV) and repetitive surges in sympathetic activity, efforts have been made to develop noninvasive tools to diagnose OSAS using HRV associated with sleep-related breathing disorders (SRBDs) [5]. In an era of continuously increasing numbers of new methods for HRV determination and ongoing discussion about the relevance of different HRV measures, the frequency component of interbeat interval increment (III) has been introduced as a computationally simple and inexpensive tool in the screening of OSAS. By applying this method, previous studies from the current authors' group demonstrated that a HRV variable named power spectral density of III of very low frequencies (\%VLFI) $[6,7]$ is a useful criterion to establish a diagnosis of OSAS. Similar results have recently been published in a chronic heart failure population [8] in that a value of \%VLFI $<2.4$ excludes obstructive events. However, since a complex interaction of upper airway obstruction, hypoxaemia and sleep fragmentation occurs during obstructive apnoea, modulation of disrupted

\section{AFFILIATIONS}

*Dept of Clinical Physiology and Exercise, Faculty of Medicine, Jacques Lisfranc University Jean Monnet, Saint-Etienne, France.

\# Sleep Laboratory, Dept of Psychiatry, University Hospital, Geneva, Switzerland.

CORRESPONDENCE

F. Roche

Physiology laboratory

EFCR

CHU Nord - Niveau 6

F - 42055 Saint-Etienne Cedex 2

France

Fax: 33477828447

E-mail: Frederic.Roche@

univ-st-etienne.fr

Received:

March 252006

Accepted after revision:

January 162007

STATEMENT OF INTEREST

None declared.
European Respiratory Journal Print ISSN 0903-1936

Online ISSN 1399-3003 
sleep [9] and hypoxaemia must also be considered in the interpretation of III in the clinical setting.

The purpose of the present study was to determine the predictive accuracy of frequency-domain parameters of the III known to accurately extract the very low frequency components, in a large group of patients referred for suspected SRBDs. The accuracy of the method was evaluated with regard to the most recent definition of SRBDs, considering the influence of comorbidities, severity of sleep fragmentation and associated vascular disease.

\section{METHODS}

\section{Study group}

Patients undergoing polysomnography (PSG) for suspected OSAS were enrolled in the study. Exclusion criteria were: 1) presence of diseases not allowing analysis of HRV $(n=25)$, i.e. permanent or paroxysmal atrial fibrillation, Shy-Drager syndrome, polyneuropathy, permanent ventricular or atrial pacing $(n=2) ; 2)$ current intake of anti-arrhythmic drugs or digitalis $(n=10)$; and 3) previous treatment for OSAS by continuous positive airway pressure therapy, surgery and/or oral device $(n=15)$. Of the initial sample, 150 patients $(118$ males, 32 females) with a mean \pm SD age of $51.3 \pm 9.7$ yrs and body mass index (BMI) of $30.4 \pm 5.3 \mathrm{~kg} \cdot \mathrm{m}^{-2}$ fulfilled the inclusion criteria.

All patients visited an experienced sleep specialist for a detailed clinical interview concerning the primary complaint motivating the consultation (i.e. snoring (42\%), reported apnoeas $(70.5 \%)$ and sleepiness $(11 \%))$ and past and present medical history, with special focus on cardiac and cerebrovascular disease, hypertension, obstructive or restrictive lung disease, metabolic disorders, psychiatric diseases and actual drug intake. Patients were informed that some of the collected data would be used for research purposes and they gave written informed consent.

\section{Nocturnal sleep studies}

PSG included seven electroencephalograms, right and left electrooculograms and one electromyogram of chin muscles for conventional sleep staging. Respiratory airflow was monitored with a nasal cannula connected to a pressure transducer (Protech2, Minneapolis, MN, USA), thoracic and abdominal respiratory movements with piezoelectric strain gauges, and tracheal sound by microphone. Arterial oxygen saturation $\left(\mathrm{Sa}_{1} \mathrm{O}_{2}\right)$ was continuously measured with a finger oximeter. Sleep was scored using the criteria of RECHTSCHAFFEN and KALES [10] for epochs of $20 \mathrm{~s}$ by a scorer experienced in the use of standard guidelines. As indices of sleep fragmentation, the number of awakenings, the number of sleep stage changes and the sleep fragmentation index (SFI) were defined [11, 12]. The SFI was calculated as the total number of sleep stage changes and awakenings lasting $20 \mathrm{~s}$ divided by total sleep time per hour [11]. Respiratory events were scored using standard criteria [13]. Hypopnoea were defined as a $\geqslant 50 \%$ reduction in airflow from the baseline value lasting $\geqslant 10 \mathrm{~s}$ and associated with $4 \%$ desaturation or an arousal. Apnoeas were defined as the absence of airflow on the nasal cannula lasting for $>10 \mathrm{~s}$. The apnoea/hypopnoea index (AHI) was established as the ratio of the number of apnoeas and hypopnoea per hour of sleep. Mean $\mathrm{Sa}_{1} \mathrm{O}_{2}$, the percentage of sleep time where $\mathrm{Sa}_{1} \mathrm{O}_{2}$ was $<90 \%$, and the minimal $\mathrm{Sa}_{1} \mathrm{O}_{2}$ value recorded during sleep were considered as indices of nocturnal hypoxaemia. Patients were classified as having OSAS if the AHI was $>15$ events $\cdot h^{-1}$ according to a previous report from the current authors' group $[6,7]$.

\section{Synchronised ECG Holter monitoring with III analysis}

Standard three-channel ECG Holter tape recorders (Vista; Novacor, Rueil-Malmaison, France) were used to acquire the data and were applied $\sim 2 \mathrm{~h}$ before the beginning of the polysomnographic recording and removed after the final awakening. The polygraphic data were matched with the ECG Holter data using polygraphic clock time synchronised with ECG Holter time before "lights out". Two independent scorers, each one blinded to the results of the other, performed the HRV analysis and the synchronised polysomnographic scoring. The sampling rate of the ECG was $200 \mathrm{~Hz}$. This ensured an accuracy of $5 \mathrm{~ms}$ for the times of the identified $\mathrm{R}$ peaks. In order to obtain the RR interval the time when each QRS occurs was determined by dedicated software (HolterSoft; Novacor). Ventricular and supraventricular ectopic beats, and artefacts defined by RR interval $>2,000 \mathrm{~ms}$, were automatically removed from analysis. The RR series were then built by concatenation of the consecutive sections of normal RR sequences. Next, a cubic spline interpolated the irregularly spaced RR series onto a regularly spaced time base (sampling rate $4 \mathrm{~Hz}$ ). It should be noted that cubic-spline interpolating smoothed the RR series and the first derivative. For the whole night recording, the following cardiac autonomic activity measures of HRV were performed according to published criteria [14] using frequency and time domain analyses. Briefly, for frequency domain analysis the following were calculated: the total power (Ptot), the very low frequency power (VLF: $0.00-0.04 \mathrm{~Hz}$ ), the low frequency power (LF: $0.04-0.015 \mathrm{~Hz}$ ) and the high frequency power (HF: $0.15-0.40 \mathrm{~Hz}$ ). LFnu and HFnu were calculated as LF and HF, respectively, divided by Ptot-VLF. The LF/HF ratio was also calculated. Overall, the VLF represents parasympathetic activity, LF and LFnu represent both sympathetic and parasympathetic activity, and HF and HFnu represent parasympathetic activity. Timedomain methods allow the assessment of the SD of the normal electrocardiographic R-R intervals, the SD of the average normal electrocardiographic R-R intervals, the square root of the mean squared differences of successive R-R intervals, the number of interval differences of successive R-R intervals (NN50) and the proportion derived by dividing NN50 by the total number of R-R intervals. To identify the very-low frequency oscillations, a power spectral analysis of III was performed, as a function of the inverse of the increment. The methodology of such an analysis has been extensively developed in previous studies [6, 7]. Briefly, III is defined by the backward difference of RR values. So, III time series was obtained straightforwardly by taking the difference between successive RR values sampling at $4 \mathrm{~Hz}$. This last process is similar to estimating the first derivative of the RR signal. To identify the very low frequency component of III variations present in the recording Fourier transform was used. The III power spectral density is estimated by dividing the III signal into successive blocks, and averaging squared-magnitude Fourier transforms of the signal blocks. The block size was 4,096 points, i.e. $\sim 1,000$ s. The present size ensures a Fourier 
frequency step which was, for the most part, smaller than the VLFI frequency range. Thus, \%VLFI was calculated as the ratio of power spectral in the very low frequency range, i.e. $0.01-$ $0.05 \cdot$ beat $^{-1}$, over the total power spectral density (0.01-0.5 beat $\left.^{-1}\right)$. The $\%$ VLFI variable has been reported as the most accurate variable for the prediction of OSAS and was the only one retained in the present study, in accordance with the results of previous studies [6].

\section{Statistical analysis}

Differences in polygraphic and clinical findings between patients without (OSAS-) and with OSAS (OSAS+) were analysed using the Mann-Whitney U-test and the Chi-squared test, respectively. To evaluate the ability of the \%VLFI to discriminate between diseased and nondiseased status, according to several AHI thresholds $(>5,>10,>15,>30$ and $>50$ events $\cdot h^{-1}$ ), receiver operating characteristic (ROC) curve analysis was used, with the areas under the curves represented by $\mathrm{W} \pm \mathrm{SE}$ as well as the calculated confidence interval (CI). ROC analysis was also used to evaluate the ability of the $\%$ VLFI to predict sleep fragmentation when an SFI $>50$ events $\cdot h^{-1}$ was considered. The highest separation power was established to obtain a threshold value with an optimised sensitivity or specificity for \%VLFI according the highest Wvalue obtained in previous studies. The predictive accuracy of the \%VLFI parameter was evaluated using classical sensitivity, specificity and predictive values according to \%VLFI threshold values obtained in previous studies. Statistical significance was determined as $\mathrm{p}<0.05$ after Bonferroni correction. Values were expressed as mean $\pm \mathrm{SD}$.

\section{RESULTS}

Table 1 shows the anthropometric and clinical characteristics of the patient groups. In total, $100(66.7 \%)$ patients were diagnosed using polysomnographic recording as having OSAS, with the remaining patients not considered to have the disease. OSAS patients were more frequently males, were found to be older and they had a higher BMI. Compared to OSAS- patients, OSAS+ patients were referred more frequently for witnessed breathing pauses during sleep, although there was no difference in the level of daytime sleepiness as a major complaint. A detailed clinical interview did not reveal significant differences between groups with regard to incidence of cardiovascular, neurological, psychiatric or metabolic disease, except for hypertension, which more frequently affected patients with OSAS. Polysomnographic data of the two groups are reported in table 2. As expected, the groups differed significantly in all parameters of sleep continuity and sleep fragmentation, with OSAS patients having lower amounts of slow-wave sleep and rapid eye movement sleep and longer time spent in sleep stage 1. Analysis of respiratory disturbances in the two groups revealed that OSAS+ patients had a higher AHI and more severe nocturnal hypoxaemia. No differences in subjective daytime sleepiness on the Epworth Sleepiness Scale were found between groups. Table 3 summarises the average value for each cardiac variable during the sleep period in both OSAS- and OSAS+ patients. When the standard HRV measures within the night were calculated for the two groups of patients, no statistically significant difference was observed. Only the frequency-domain III parameter (\%VLFI) was found to be significantly higher in patients with

\begin{tabular}{|c|c|c|c|}
\hline \multirow[t]{2}{*}{ TABLE 1} & \multicolumn{3}{|c|}{$\begin{array}{l}\text { Anthropometric and clinical data of patients } \\
\text { without and with obstructive sleep apnoea/ } \\
\text { hypopnoea syndrome (OSAHS) }\end{array}$} \\
\hline & OSAHS- & OSAHS+ & p-value \\
\hline Subjects $n$ & 50 & 100 & \\
\hline Age yrs & $48.5 \pm 8.5$ & $52.8 \pm 10.0$ & 0.03 \\
\hline BMI $\mathbf{k g} \cdot \mathbf{m}^{-2}$ & $29.5 \pm 6.0$ & $30.9 \pm 4.9$ & 0.04 \\
\hline Neck circumference $\mathrm{cm}$ & $39.9 \pm 3.6$ & $42.1 \pm 3.4$ & 0.02 \\
\hline $\begin{array}{l}\text { Epworth Sleepiness Scale } \\
\text { score }\end{array}$ & $10.3 \pm 5.1$ & $9.5 \pm 4.9$ & NS \\
\hline \multicolumn{4}{|l|}{ Primary complaints $\%$} \\
\hline Snoring & 68 & 40 & $<0.02$ \\
\hline Apnoea & 16 & 39 & 0.003 \\
\hline Sleepiness & 8 & 12 & NS \\
\hline \multicolumn{4}{|l|}{ History \% } \\
\hline Hypertension & 24 & 40 & 0.04 \\
\hline Cardiovascular disease & 4 & 6 & NS \\
\hline Diabetes & 4 & 9 & NS \\
\hline Hypercholesterolemia & 22 & 31 & NS \\
\hline ACE inhibitors & 10 & 30 & 0.02 \\
\hline$\beta$-Blockers & 7 & 15 & NS \\
\hline
\end{tabular}

Data are presented as mean $\pm S D$, unless otherwise stated. BMI: body mass index; ACE: angiotensin-converting enzyme; NS: nonsignificant. p-values reflect differences between patients without and with OSAHS (Chi-squared and MannWhitney U-test).

TABLE 2 Polysomnographic data of the population

\begin{tabular}{|c|c|c|c|}
\hline & OSAHS- & OSAHS+ & p-value \\
\hline Subjects $\mathrm{n}$ & 50 & 100 & \\
\hline TST min & $435.9 \pm 49.5$ & $417.8 \pm 78.6$ & NS \\
\hline Stage $1 \mathrm{~min}$ & $55.5 \pm 22.5$ & $80.4 \pm 32.6$ & $<0.0001$ \\
\hline Stage $2 \mathrm{~min}$ & $225.5 \pm 54.0$ & $232.2 \pm 63.9$ & NS \\
\hline Stage $3 \mathrm{~min}$ & $59.6 \pm 31.7$ & $39.8 \pm 30.8$ & 0.001 \\
\hline Stage $4 \mathrm{~min}$ & $15.4 \pm 19.7$ & $7.5 \pm 13.8$ & 0.006 \\
\hline Stage REM min & $80.1 \pm 28.8$ & $58.0 \pm 31.5$ & $<0.0001$ \\
\hline WASO min & $69.5 \pm 43.2$ & $106.6 \pm 64.3$ & 0.001 \\
\hline SE \% & $83.8 \pm 8.4$ & $77.2 \pm 12.8$ & 0.002 \\
\hline SFI events $\cdot h^{-1}$ & $42.5 \pm 14.3$ & $75.1 \pm 44.1$ & $<0.0001$ \\
\hline PLMS index $h^{-1}$ & $9.5 \pm 19.1$ & $12.0 \pm 16.4$ & NS \\
\hline$A H I h^{-1}$ & $7.7 \pm 3.8$ & $45.6 \pm 23.6$ & $<0.0001$ \\
\hline Minimum $\mathrm{Sa}, \mathrm{O}_{2} \%$ & $83.9 \pm 4.4$ & $77.4 \pm 8.9$ & $<0.0001$ \\
\hline Mean $\mathrm{Sa}, \mathrm{O}_{2} \%$ & $93.3 \pm 1.6$ & $92.0 \pm 3.0$ & 0.01 \\
\hline$\%$ time $\mathrm{Sa}, \mathrm{O}_{2}<\mathbf{9 0 \%}$ & $7.2 \pm 12.2$ & $19.9 \pm 24.1$ & $<0.0001$ \\
\hline ODI $h^{-1}$ & $4.8 \pm 4.4$ & $28.4 \pm 19.9$ & $<0.0001$ \\
\hline Snoring index $\mathrm{h}^{-1}$ & $335.0 \pm 322.6$ & $409.1 \pm 219.5$ & 0.04 \\
\hline
\end{tabular}

Data are presented as mean $\pm \mathrm{SD}$, unless otherwise stated. OSAHS: obstructive sleep apnoea/hypopnoea syndrome; TST: total sleep time; REM: rapid eye movement; WASO: wake after sleep onset; SE: sleep efficiency; SFI: sleep fragmentation index; PLMS: periodic limb movements in sleep; AHI: apnoea/ hypopnoea index; $\mathrm{Sa}_{1} \mathrm{O}_{2}$ : arterial oxygen saturation; ODI: oxygen desaturation index. p-values reflect differences between patients with and without OSAHS (Mann-Whitney U-test). 


\begin{tabular}{|c|c|c|c|}
\hline \multirow[t]{2}{*}{ TABLE 3} & \multicolumn{3}{|c|}{$\begin{array}{l}\text { Selected frequency domain measures of heart } \\
\text { rate variability during sleep in patients without } \\
\text { and with obstructive sleep apnoea/hypopnoea } \\
\text { syndrome (OSAHS) }\end{array}$} \\
\hline & OSAHS- & OSAHS+ & $\mathrm{p}$-value \\
\hline Subjects $\mathrm{n}$ & 50 & 100 & \\
\hline RR mean ms & $878.5 \pm 113.9$ & $919.4 \pm 117.7$ & NS \\
\hline NN50 n & $3977.7 \pm 4053.4$ & $4073.5 \pm 3527.7$ & NS \\
\hline pNN50 \% & $7.2 \pm 6.1$ & $8.3 \pm 7.7$ & NS \\
\hline SDANN ms & $76.4 \pm 33.6$ & $79.4 \pm 28.2$ & NS \\
\hline SDNN ms & $97.1 \pm 35.1$ & $103.4 \pm 31.0$ & NS \\
\hline SDNN index ms & $44.1 \pm 14.2$ & $49.3 \pm 17.7$ & NS \\
\hline RMSSD ms & $34.3 \pm 23.1$ & $33.7 \pm 14.8$ & NS \\
\hline Ptot $\mathrm{ms}^{2}$ & $2248.3 \pm 1229.4$ & $3043.2 \pm 2304.0$ & NS \\
\hline VLF $\mathrm{ms}^{2}$ & $1328.2 \pm 5670.8$ & $7157.8 \pm 5171.1$ & 0.05 \\
\hline LF $\mathrm{ms}^{2}$ & $525.8 \pm 326.9$ & $651.8 \pm 505.0$ & NS \\
\hline $\mathrm{HF} \mathrm{ms}^{2}$ & $261.3 \pm 241.2$ & $288.3 \pm 231.8$ & NS \\
\hline LFnu & $79.8 \pm 1.7$ & $65.4 \pm 11.6$ & NS \\
\hline HFnu & $42.8 \pm 783.4$ & $34.6 \pm 11.6$ & NS \\
\hline LF/HF ratio & $3.40 \pm 2.2$ & $3.45 \pm 2.1$ & NS \\
\hline$\%$ VLFI & $3.53 \pm 2.0$ & $5.38 \pm 2.0$ & $<0.0001$ \\
\hline \multicolumn{4}{|c|}{$\begin{array}{l}\text { Data are presented as mean } \pm \text { SD. RR mean: mean of the NN intervals; NN50: } \\
\text { numbers of pairs of adjacent NN intervals differing by }>50 \text { ms; pNN50: NN50 } \\
\text { count divided by the total number of all NN intervals; SDANN: SD of the } \\
\text { averages of the NN intervals in all } 5 \text { min segments of the entire period; SDNN: } \\
\text { SD of all NN intervals; SDNN index: mean of the SD of all NN intervals; RMSSD: } \\
\text { square root of the mean of the squares of differences between adjacent NN } \\
\text { intervals; Ptot: total power; VLF: power at very low frequency; LF: power at low } \\
\text { frequency; HF: power at high frequency; LFnu: LF power in normalised units } \\
\text { (power spectrum/Ptot-VLF } \times 100 \text { ); HFnu: HF power in normalised units (power } \\
\text { spectrum/Ptot-VLF } 100 \text { ); \%VLFI: ratio of power spectral density in the very low } \\
\text { frequency range over the total power spectral density; NS: nonsignificant. } \\
\text { p-values reflect the differences between patients without and with OSAHS } \\
\text { (Mann-Whitney U-test). }\end{array}$} \\
\hline
\end{tabular}

OSAS $(5.38 \pm 2.04$ versus $3.53 \pm 2.03 \% ; \mathrm{p}<0.0001)$. A weak but significant linear correlation was found between $\mathrm{AHI}$ and $\%$ VLFI $\left(\mathrm{R}^{2}=0.10 ; \mathrm{p}<0.001\right)$. A ROC curve (continuous data) built for \%VLFI confirmed that this variable was able to separate OSAS+ from OSAS- status with statistical significance $(\mathrm{W}=0.70 ; \mathrm{p}<0.0001 ;$ fig. 1$)$ and to predict sleep fragmentation (fig. 2) according to an SFI $\geqslant 50$ events $\cdot h^{-1} \quad(W=0.68$; $\mathrm{p}<0.0001)$. In order to determine if severity of disease affects the ability of \%VLFI to discriminate AHI values, ROCs were built according to AHI threshold. As summarised in table 4, $\%$ VLFI appeared to be more efficient in predicting moderateto-severe OSAS, as $\mathrm{W}$-values were higher when the AHI was $>15$ events $\cdot \mathrm{h}^{-1}$. Using a \%VLFI threshold of 2.4 , sensitivity for OSAS prediction was $91 \%$, specificity $34 \%$, positive predictive value $73.4 \%$ and negative predictive value $65.4 \%$ (true negatives: 17; false negatives: 9; true positives: 91; false positives: 33 patients). Using a \%VLFI threshold of 4 , sensitivity for OSAS prediction was $64 \%$, specificity $69.2 \%$, positive predictive value $80 \%$ and negative predictive value 48.6\% (true negatives: 34 ; false negatives: 36 ; true positives: 64 ; false positives: 16). Among the false-positives group, an enhanced SFI $\left(45.8 \pm 14.4\right.$ events $\left.\cdot h^{-1}\right)$ was found. SFI exceeded

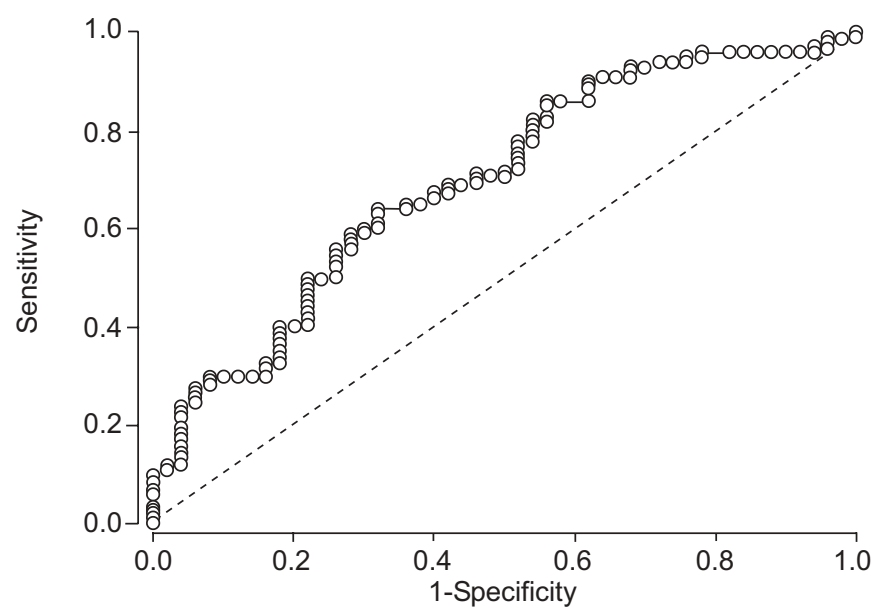

FIGURE 1. Receiver operating characteristic curve for the prediction of obstructive apnoea/hypopnoea syndrome (apnoea/hypopnoea index $\geqslant 15$ events $\cdot h^{-1}$ ) from the power spectral density of interbeat interval increment of very low frequencies. Area under the curve $=0.70$

50 events $\cdot \mathrm{h}^{-1}$ in 16 cases reporting insomnia and in 28 cases having periodic limb movements in sleep (PLMS) independent of respiratory events (mean PLMS index: 28.4 \pm 15 ). Using multiple logistic regression analysis, after adjustment for age, sex and neck circumference, OSAS status (AHI $\geqslant 15$ events $\cdot h^{-1}$, odds ratio (OR): 2.6; 95\% CI: 1.2-5.8; Chi-squared $=6.65$; $\mathrm{p}=0.009)$ and sleep fragmentation as indicated by an SFI $\geqslant 50$ events $\cdot h^{-1}$ (OR: 2.3; 95\% CI: 1.3-5.2; Chi-squared $=10.2$, $\mathrm{p}=0.0014$ ) appeared to be independently and significantly associated with $\%$ VLFI $>4$.

\section{DISCUSSION}

With both sleep quality and respiratory disorders taken into account, the present study demonstrates new findings in addition to previous observations of III patterns in patients with SRBDs. The results presented support the existence of differences in autonomic control in patients with OSAS, with VLF and \%VLFI being significantly higher in patients with

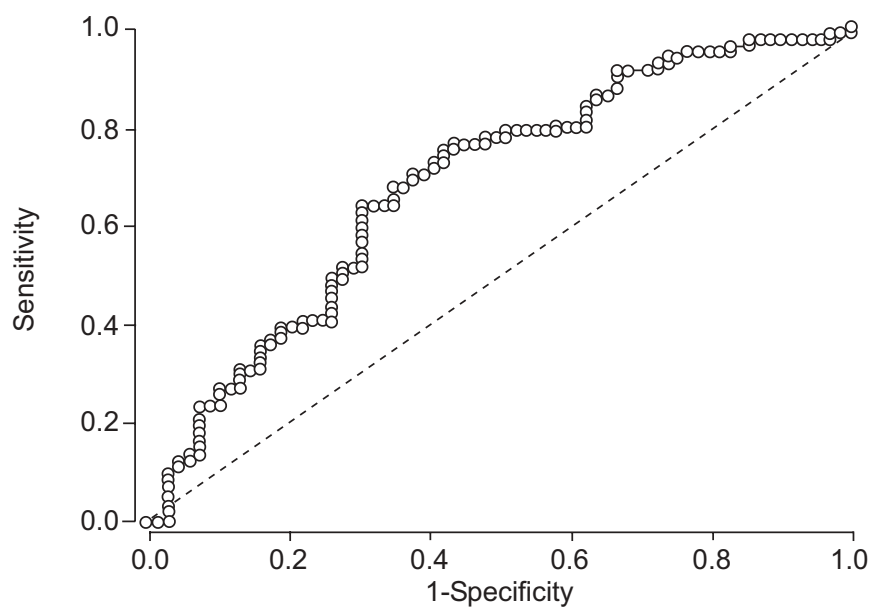

FIGURE 2. Receiver operating characteristic curve associating the power spectral density of interbeat interval increment of very low frequencies with sleep fragmentation index $\geqslant 50$ events $\cdot h^{-1}$. Area under the curve $=0.68$ 


\begin{tabular}{|c|c|c|c|}
\hline TABLE 4 & \multicolumn{3}{|c|}{$\begin{array}{l}\text { Evaluation of the power spectral density of } \\
\text { interbeat interval increment of very low } \\
\text { frequencies in predicting obstructive sleep } \\
\text { apnoea/hypopnoea syndrome according to } \\
\text { several apnoea/hypopnoea index (AHI) } \\
\text { thresholds using receiver operating } \\
\text { characteristic curve analysis }\end{array}$} \\
\hline $\begin{array}{l}\text { AHI threshold } \\
\text { events } \cdot h^{-1}\end{array}$ & W-value & $95 \% \mathrm{Cl}$ & $p$-value \\
\hline 5 & $0.65 \pm 0.05$ & $0.55-0.75$ & 0.007 \\
\hline 10 & $0.65 \pm 0.05$ & $0.57-0.74$ & 0.001 \\
\hline 15 & $0.70 \pm 0.04$ & $0.61-0.79$ & $<0.0001$ \\
\hline 30 & $0.69 \pm 0.05$ & $0.59-0.79$ & 0.0006 \\
\hline 50 & $0.71 \pm 0.06$ & $0.59-0.84$ & 0.0005 \\
\hline
\end{tabular}

moderate-to-severe OSAS. Nevertheless, also considering hypoxaemia and SFI instead of only analysing AHI, the results suggest that differences in HRV are not of the same magnitude within patients. Independent of the frequency of sleepdisordered breathing, a trend towards increased sympathetic activity is observed when sleep is more fragmented. Indeed, the current authors suggest that determination of HRV may be affected by concomitant sleep disorders which induce sleep fragmentation and sleep discontinuity. These results stress the importance of considering the relationship between OSAS and sleep fragmentation when HRV measurement is considered as a clinical tool in the diagnosis of SRBDs.

The main goal of the present study was to characterise the changes in HRV measurements during sleep in patients with sleep-disordered breathing, in order to establish if these measures may indicate the occurrence of OSAS. Here, a novel method for the detection of SRBDs was presented, which overcomes the shortcomings of spectral ECG analysis. The first important finding of the present study was that while standard time- and frequency-domain parameters were less sensitive to modification of autonomic control in OSAS, \%VLFI appears to be a more sensitive tool for the detection of not only the autonomic changes associated with apnoea but also the severity of the disease. The current authors confirm, in a larger population, the high sensitivity and negative predictive value of a low \%VLFI $(<2.4 \%)$ in excluding OSAS and the high positive predictive value of $\%$ VLFI $>4 \%$ in confirming diagnosis. Using the same \%VLFI threshold, similar results have been obtained in patients with central sleep apnoea [8] in which a $\%$ VLFI $<2.4$ ruled out association with OSAS with a specificity of $65 \%$ and a sensitivity of $85 \%$. For a \%VLFI threshold of 2.4, sensitivity for OSAS prediction was $91 \%$ but specificity $34 \%$, indicating concomitant sleep alteration affecting HRV. In contrast, using a \%VLFI threshold of 4, sensitivity for OSAS prediction was $64 \%$ and specificity $69.2 \%$. Thus, frequency-domain analysis of HRV could be considered to be a convenient method of quantifying autonomic changes taking place during apnoeas.

Despite the fact that there is wide evidence that HRV reflects changes in the autonomic system related to apnoea, it is less clear whether the rise in sympathetic activity reflects only the apnoea effect or if it is also affected by other factors, such as hypoxaemia and sleep fragmentation, both influencing the autonomic system during sleep. The results of the present study showed that the magnitude of HRV changes were affected by sleep fragmentation, since a greater \%VLFI was found in patients with a higher SFI. Previous studies have shown that the analysis of several autonomic changes occurring during sleep could be applied to detect sleep fragmentation in patients with sleep disorders, e.g. pulse transit time alterations [15-17], variation in tonometry and blood pressure [18], all of which were found to reflect sleep discontinuity and sleep fragmentation. The data arising from the present study, despite the fact that SFI is a simple estimate of sleep fragmentation, indicate that the analysis of HRV could be used as a surrogate of sleep quality, because \%VLFI is greater when sleep fragmentation and discontinuity are higher. Compared with other methods, the III methodology represents a simpler and inexpensive tool for the quantification of autonomic nervous system activation when sleep is fragmented and disrupted [19], in relation to spontaneous or evoked arousing stimuli $[9,20-22]$. This result has important implications, from a clinical point of view, in the diagnosis of sleep disorders. Although requiring replication with a much larger patient group and with a more carefully defined sleep fragmentation, such as via microarousal index, these data implicate that increased \%VLFI may indicate both OSAS and severe sleep fragmentation. Therefore, the relative lack of specificity of the present methodology for the diagnosis of related sleep breathing disorders when concomitant sleep disorders, i.e. insomnia, restless legs syndrome and periodic leg movements, are present. This would also suggest that III may be not a potential screening tool for SRBDs when clinical history is uncertain and when the association of other sleep disorders is postulated.

The current study presents some methodological limitations that need to be considered in the discussion of the results. First, the sample represents a fairly homogeneous apnoeic population which was, for the most part, referred for snoring and apnoea. As a result, this precludes generalisation to patients with other sleep disorders, and the application of such a described algorithm of III in a general population could result in a significant alteration of the predictive value of the \%VLFI. Only a large study comparing \%VLFI with conventional PSG realised in a middle aged population with different sleep disorders could help to affirm the role of III in the clinical screening of OSAS. Secondly, the threshold used to dichotomise OSAS was more stringent than the usual AHI $>5$ events $\cdot h^{-1}$ criterion, and thus limits the interpretation of $\%$ VLFI as a screening tool for SRBDs, \%VLFI being, in the present population, a useful marker to diagnose moderate-tosevere cases. It would be of interest in subsequent studies to evaluate whether III may diagnose the full spectrum of sleepdisordered breathing, including upper airway resistance syndrome and simple snoring. Finally, some discrepancy in the cut-off values ruling out OSAS were found in this population compared with previous studies [6], with a \%VLFI $<2.4$ less specific for the diagnosis of moderate-to-severe cases. Hourly \%VLFI appeared to be predictor of AHI and the prediction of an AHI $>20$ events $\cdot h^{-1}$ was more accurate for a 
$\%$ VLFI value $\geqslant 4 \%$. Such threshold values could be appropriate in previous studies, it has been shown that positive predictive accuracy could reach $80 \%$ in a high-risk population [7].

In conclusion, the described method of ECG variability provides a general framework for the detection and characterisation of ECG variability during obstructive apnoea and hypopnoeas. Although this aim can be achieved by other methods such as pulse transit time [17] and tonometry [18], the present method has the advantage that the power spectral density of interbeat interval increment of very low frequencies obtained by simple noninvasive ECG recording predicts the recognition of sleep apnoea/hypopnoea syndrome in two thirds of the population. Combination of interbeat interval increment criteria (as a quantitative method of pulse rate rise [23] after respiratory event) and oximetry could represent a simple tool for the screening of obstructive sleep apnoea syndrome [24]. Therefore, interbeat interval increment seems to be a useful, simple and novel measure that could allow more clinicians to identify patients with suspected sleep apnoea. However, since greater power spectral density of interbeat interval increment of very low frequencies may be found when sleep is more fragmented, other sleep disorders could be suspected when this variable is increased, emphasising the need to propose in-laboratory studies when other sleep disorders are hypothesised by clinical history. Future studies including larger sample of patients with different sleep disorders are needed to identify patterns of heart rate variability in terms of diagnosis, clinical features and degree of sleep alterations.

\section{ACKNOWLEDGEMENTS}

The authors are indebted to O. Bigaignon, N. Grazian, J-R. Borderies, G. Ascher (all Novacor Rueil Malmaison, France) and V. Dauphinot (Cetaf, Saint Etienne, France) for their assistance with the study.

\section{REFERENCES}

1 Young T, Palta M, Dempsey J, Skatrud S, Weber S, Badr S. The occurrence of sleep-disordered breathing among middle-aged adults. N Engl J Med 1993; 328: 1230-1235.

2 Barbé F, Pericas J, Munoz A, Findley L, Anto JM, Agusti AGN. Automobile accidents in patients with sleep apnoea syndrome. An epidemiological and mechanistic study. Am J Respir Crit Care Med 1998; 158: 18-22.

3 He J, Kryger MH, Zorick FJ, Conway W, Roth T. Mortality and apnea index in obstructive sleep apnea. Experience in 385 male patients. Chest 1988; 94: 9-14.

4 Lavie P, Herer P, Peled R, et al. Mortality in sleep apnea patients: a multivariate analysis of risk factors. Sleep 1995; 18: 149-157.

5 Roche F, Pichot V, Sforza E, et al. Predicting sleep apnoea syndrome from heart period: a time-frequency wavelet analysis. Eur Respir J 2003; 22: 937-942.

6 Roche F, Duverney D, Court-Fortune I, et al. Cardiac interbeat interval increment for the identification of obstructive sleep apnoea. Pacing Clin Electrophysiol 2002; 25: 1192-1199.

7 Roche F, Sforza E, Duverney D, et al. Heart rate increment: an electrocardiological approach for the early detection of obstructive sleep apnoea/hypopnoea syndrome. Clin Sci (Lond) 2004; 107: 105-110.
8 Vazir A, Dayer M, Hastings PC, et al. Can heart rate variation rule out sleep-disordered breathing in heart failure? Eur Respir J 2006; 27: 571-577.

9 Trinder J, Allen N, Kleiman J, et al. On the nature of cardiovascular activation at an arousal from sleep. Sleep 2003; 26: 543-551.

10 Rechtschaffen A, Kales A. eds, A Manual of Standardized Terminology, Technique and Scoring System for Sleep Stages of Human Sleep. Washington, US Government Printing Office, 1968.

11 Haba-Rubio J, Ibanez V, Sforza E. An alternative measure of sleep fragmentation in clinical practice: the sleep fragmentation index. Sleep Med 2004; 5: 577-581.

12 EEG arousals: scoring rules and examples: a preliminary report from the Sleep Disorders Atlas Task Force of the American Sleep Disorders Association. Sleep 1992; 15: 173-184.

13 Sleep-related breathing disorders in adults: recommendations for syndrome definition and measurement techniques in clinical research. The Report of an American Academy of Sleep Medicine Task Force. Sleep 1999; 22: 667-689.

14 Heart rate variability: standards of measurements, physiological interpretation and clinical use. Task Force of the European Society of Cardiology and the North American Society of Pacing and Electrophysiology. Circulation 1996; 93: 1043-1065.

15 Stradling JR, Davies RJO, Pitson DJ. New approaches to monitoring sleep-related breathing disorders. Sleep 1996; 19: Suppl. 9, S77-S84.

16 Pitson DJ, Stradling JR. Value of beat-to-beat blood pressure changes, detected by pulse transit time, in the management of the obstructive sleep apnoea/hypopnoea syndrome. Eur Respir J 1998; 12: 685-692.

17 Levy P, Pepin JL. Sleep fragmentation: clinical usefulness of autonomic markers. Sleep Med 2003; 4: 489-491.

18 Pillar G, Bar A, Shilner A, Schnall R, Lavie P. Autonomic arousal index: an automated detection based on peripheral arterial tonometry. Sleep 2001; 25: 543-549.

19 Morrell MJ, Finn L, Kim H, Peppard PE, Badr MS, Young T. Sleep fragmentation, awake blood pressure, and sleepdisordered breathing in a population-based study. Am J Respir Crit Care Med 2000; 162: 2091-2096.

20 Catcheside PG, Chiong SC, Mercer J, Saunders NA, McEvoy RD. Noninvasive cardiovascular markers of acoustically induced arousal from non-rapid-eye-movement sleep. Sleep 2002; 25: 797-804.

21 Sforza E, Juony C, Ibanez V. Cardiac activation during arousals in humans: further evidence for hierarchy in the arousal response. Clin Neurophysiol 2000; 111: 1611-1619.

22 Sforza E, Pichot V, Barthélémy JC, Haba-Rubio J, Roche F. Cardiovascular variability during periodic leg movements: a spectral analysis approach. Clin Neurophysiol 2005; 116 : 1096-1104.

23 Adachi H, Mikami A, Kumano-go T, et al. Clinical significance of pulse rate rise during sleep as a screening marker for the assessment of sleep fragmentation in sleepdisordered breathing. Sleep Med 2003; 4: 537-542.

24 Raymond B, Cayton RM, Chappell MJ. Combined index of heart rate variability and oximetry in screening for the sleep apnoea/hypopnoea index syndrome. J Sleep Res 2003; 12: 53-61. 